\title{
Numerical Modeling of Tidal Effects and Hydrodynamics in the Po River Estuary
}

\author{
Célestin Leupi ${ }^{1}$, Michel Deville ${ }^{1}$, and Mustafa Siddik Altinakar ${ }^{2}$ \\ ${ }^{1}$ ISE-STI-LIN, Ecole Polytechnique Fédérale, \\ Station 9, Lausanne 1015, Switzerland \\ Tel.: +41.21.693.25.07; Fax: +41.21.693.36.46 \\ celestin.leupi@epfl.ch \\ Tel.: +41.21.693.53.18; Fax: + 41.21.693.36.46 \\ michel.deville@epfl.ch \\ 2 NCCHE, The University of Mississipi, \\ Carrier Hall Room 102 University, \\ MS 38677 USA
}

\begin{abstract}
The present work contributes to the numerical simulation of complex turbulent multiphasic fluid flows encountered in estuarine channels. A numerical solution is based on Reynolds averaged NavierStokes equations using the mass preserving model based on the so-called Raviart-Thomas finite element on the unstructured mesh in the horizontal plane. In the vertical, the computational domain is divided into number of layers at predefined heights and the method uses a conventional conforming finite element scheme, with the advantage that the lowermost and uppermost layers variable height allow a faithful representation of the time-varying bed and free surface, respectively. A robust up-to-date algorithm is used for computing the eddy viscosity from the efficient $k-\varepsilon$ turbulence model for variable density fluid flows. Finally, the capability and the predicting performance of the model are successfully evaluated by applying it to the simulation of the Po River Estuary (PRE) in Italy.
\end{abstract}

Keywords: Finite Element model; multiphasic fluid flows; $k-\varepsilon$ turbulence model; multi-layers system; unstructured grid; Estuary.

\section{Introduction}

Estuaries, where are situated many famous ports of the world, are the meeting point between the freshwater charged with sediment from river and the saline sea cold water. The present research finds its motivation in the increasing need for efficient management of estuaries by taking into account the various conflicting environmental and socio-economical aspects.

Shallow water equations have been successfully applied to many engineering problems and their use has become common practice in environmental impact studies in estuarial and coastal regions. 
Chen et al. 3] have successfully applied a three-dimensional finite difference model with hydrostatic pressure distribution for simulating the Pearl River Estuary. Casulli and Chen [1] have developed a semi-implicit finite difference scheme for the the $3 D$ shallow water equations (SWE). Lie and Mellor [8], 9] have applied a $3 D$ finite difference model for simulation the Hudson-Raritan Estuary with a second order turbulence-closure sub-model. Lu and Wai [10] developed an efficient operator splitting scheme for 3D hybrid hydrodynamic model using the so-called sigma transformation in the vertical direction. Chau and Jiang [2] have developed a model based on POM (Princeton Ocean Model) with an orthogonal curvilinear coordinate in the horizontal direction and sigma coordinate in the vertical direction for simulating the Pearl River Estuary.

Most of the existing models use a finite difference or finite volume approach based on a topographically conform, sigma-transformed grid which, regardless of the depth, forces the same number of divisions in the water column. However, these models cannot fit to complicated geometry and this may induce large large errors if applied to complex boundary configurations.

In this study, the hydrodynamics solutions are obtained by solving the shallow water equations using implicit time marching scheme (see Leupi et al. [7], 6]), while discretization is conducted using Euler or Runge-Kutta scheme to obtain a set of algebraic equations. The model uses hydrostatic pressure distribution, and an efficient fractional time-step scheme of Mohammadi and Pironneau [12 for the $k-\varepsilon$ turbulence closure. This paper deals with the simulation of the 3D turbulent flow in the Po River Estuary in Italy.

\section{Mathematical Models}

A brief description of the model is provided in [7], 5]. Let us consider an incompressible fluid body in a three-dimensional time varying domain $\widehat{\Omega}$. Let $\Omega$ the projection of $\widehat{\Omega}$ on the $x y$ horizontal plane, bounded by the free-surface $\Gamma_{s}$ given by $z=\eta(x, y, t)$, the bottom topography $\Gamma_{b}$ given by $z=-\mathfrak{h}(x, y)$, the open boundary denoted by $\Gamma_{o}$. Where $\mathfrak{h}(x, y)$ is the distance between the bottom and the reference plane $x y$ and $\eta(x, y, t)$ the elevation of the free-surface with the respect to the reference plane $x y$.

The RANS description of the turbulent motion relies on the Boussinesq approximation. For $t>0$ and $(x, y, z) \in \widehat{\Omega}(t)$, and using the Boussinesq assumption for the hydrostatic approximation we have that

$$
\frac{1}{\rho} \frac{\partial p}{\partial z}=-g \Rightarrow p=p_{0}+\rho g(\eta-z)
$$

where $p_{0}$ is the atmospheric pressure.

For a variable density fluid under the hydrostatic assumption the system of the shallow water equations for long waves (vertical scale is negligible compared to the horizontal one) reads 


$$
\left\{\begin{array}{l}
\nabla_{x y} \cdot \mathbf{U}+\frac{\partial w}{\partial z}=0 \\
\frac{D \mathbf{U}}{D t}-\frac{\partial}{\partial z}\left(\nu_{T} \frac{\partial \mathbf{U}}{\partial z}\right)+g \nabla \eta+g \nabla\left(\int_{z}^{\eta} \frac{\Delta \rho}{\rho_{0}} \mathrm{~d} z\right)=\mathbf{F}_{x y}, \\
\frac{\partial \eta}{\partial t}+\nabla \cdot \int_{-h}^{\eta} \mathbf{U} \mathrm{d} z=\tilde{F}_{b}, \\
\frac{D \zeta}{D t}-\nabla \cdot\left[c_{\zeta} \frac{k^{2}}{\varepsilon} \nabla \zeta\right]=F_{\zeta} \quad \text { for } \quad \forall \zeta=(k, \varepsilon) \\
\frac{D \Phi}{D t}-\nabla x y\left(\nu_{T} \nabla \Phi\right)-\frac{\partial}{\partial z}\left(\nu_{T} \frac{\partial \Phi}{\partial z}\right)=F_{\Phi}, \quad \Phi=(T, S), \\
\rho=\rho(T, S),
\end{array}\right.
$$

where the unknowns are $\boldsymbol{v}, w, \eta, k, \varepsilon, T, S . \mathbf{U}=(u, v)^{T}$ is the horizontal velocity vector, $\mathbf{F}_{x y}=(\mathrm{f} v,-\mathrm{f} u)^{T}$ is vector of body forces with $f$ the Coriolis parameter, $g$ is the gravitational acceleration, $\nu_{T}$ is the eddy viscosity. $\nabla \cdot$ is the $3 D$ divergence operator, $\frac{D}{D t}$ represents the material derivative, and $\nabla_{x y}$. is the surface divergence operator. $\rho, \rho_{0}$ are respectively the fluid density and the basic water density. $\mathbf{n}_{x y}, n_{z}$ are respectively the horizontal and vertical components of the normal vector $\mathbf{n} . \boldsymbol{\Phi}(S, T)$ stands for the passive scalars: salinity (S), temperature (T) (for which $S_{0}$ and $T_{0}$ stand for the initial salinity and temperature).

The motion of the free surface is described by an alternative form of the equation, third equation of system (2), which is obtained by integrating the first equation [i.e. (local) mass balance] along the vertical coordinate by applying the Leibniz rule with the suitable kinematic boundaries conditions at the free-surface and bottom.

In the present work the state-of-the-art modeling of the $k-\varepsilon$ turbulence (fifth equation of system (2) is introduced to account for boththe stratified flows. The turbulence equations read, (see [12]).

$$
\begin{gathered}
\frac{D k}{D t}-\nabla \cdot\left[c_{\mu} \frac{k^{2}}{\varepsilon} \nabla k\right]=c_{\mu} \frac{k^{2}}{\varepsilon} G-\varepsilon-\lambda_{T} N^{2} \\
\frac{D \varepsilon}{D t}-\nabla \cdot\left[c_{\varepsilon} \frac{k^{2}}{\varepsilon} \nabla \varepsilon\right]=c_{1} k G-\frac{\varepsilon}{k}\left[c_{3} \lambda_{T} N^{2}+c_{2} \varepsilon\right]
\end{gathered}
$$

The squared shear frequency or production term $G$ is (see [5]):

$$
G=\frac{1}{2}\left(\|\nabla \mathbf{U}\|+\|\nabla \mathbf{U}\|^{T}\right)^{2}
$$

where $\|$.$\| represents the Euclidian norm, \mathbf{U}=\mathbf{U}(u, v, w)$ is the $3 \mathrm{D}$ velocity vector. 
The model constants are given: $c_{1}=0.126, c_{2}=1.92, c_{\mu}=0.09, c_{\varepsilon}=0.07$. $N$ is the Brunt-Väisälä frequency, with the related following squared buoyancy $N^{2}$ expression,

$$
N^{2}=-\frac{\nu_{T}}{\sigma_{t}} \frac{g}{\rho_{0}} \frac{\partial \rho}{\partial z}
$$

Here $\sigma_{t}=1$ is a constant, and the following expressions of eddy coefficients $\nu_{T}, \lambda_{T}$ should include

$$
\nu_{T}=S_{u} \frac{k^{2}}{\varepsilon}+\nu \quad, \quad \lambda_{T}=S_{b} \frac{k^{2}}{\varepsilon}+\lambda_{b}
$$

where $\sigma_{t}=1$ is a constant. $S_{u}$ and $S_{b}$ are the stability parameters to account for the turbulence damping in the stratified fluid flows (see [5], Luyten et al. [1] ).

In the system 2, the state equation for the variable density can be written as follows,

$$
\rho=\alpha\left(S-S_{0}\right)-\beta\left(T-T_{0}\right)
$$

where $\alpha$ and $\beta$ are the known coefficients, $\rho$ is the pore water density.

The equilibrium assumption is adopted at the bed (i.e local balance between production of turbulent kinetic energy and the rate of dissipation) and the wall functions are applied to relate the velocity to the bed shear velocity (see [7], [5]). The physical $3 D$ domain is embedded in a parallelepiped composed of $\mathcal{N}$ layers and the refine description can be found in [6]. The horizontal components of the velocity vector are defined at the middle of edges of the triangular mesh elements, while the vertical component is associated with the lower horizontal faces of the element. The horizontal velocity is approximated combining the lowest order Raviart-Thomas element $\left(\mathbb{R T}_{0}\right)$ in $x y$ plane with the $\mathbb{P}_{1}$ elements along the vertical direction.

The Lagrange-Galerkin (or characteristics Galerkin) approach is used to discretize the convection terms, while the Euler scheme or more accurate RungeKutta is used to obtain the algebraic system. The fractional time-step scheme from Mohammadi and Pironneau ([7], [12]), is adopted to solve turbulence equations. Consequently, the positivity of $k$ and $\varepsilon$ is preserved as well as the stability of the scheme (see [7]).

\section{Application to the Po River Estuary in Italy}

An efficient decoupling scheme is employed to solve hydrodynamic, turbulence, heat and salinity. The decoupling method partitions a time step into fractional steps according to physical phenomena. In the first step hydrodynamics is solved, followed by turbulence closure, and finally the advection-diffusion equations for passive scalars (temperature, salinity) are solved. PRE is located in the east of the Adriatic Sea along the direction North West-North East (NW-NE). The axis of PRE is east longitude $12.05^{\circ}$ and north latitude $44^{\circ} 96667$. The $M 2$ main 
tidal component is taken into account in this study and the mean wind speed is $30-50[\mathrm{~km} / \mathrm{h}]$. The average discharge of the PRE is $1.5 \times 10^{3}\left[\mathrm{~m}^{3} / \mathrm{s}\right]$. For simulating the tidal currents and sediment transport in PRE, a large area was selected and the 2D horizontal plane mesh. The north upstream boundary has been set far upstream the five open boundaries. The available field data for the model calibration were mostly provided by the MOX (Modeling and Scientific Computing), Department of Mathematics, Politecnico of Milano and the Istituto di ricerca Sulle Acque, CNR, Milan, Italy.
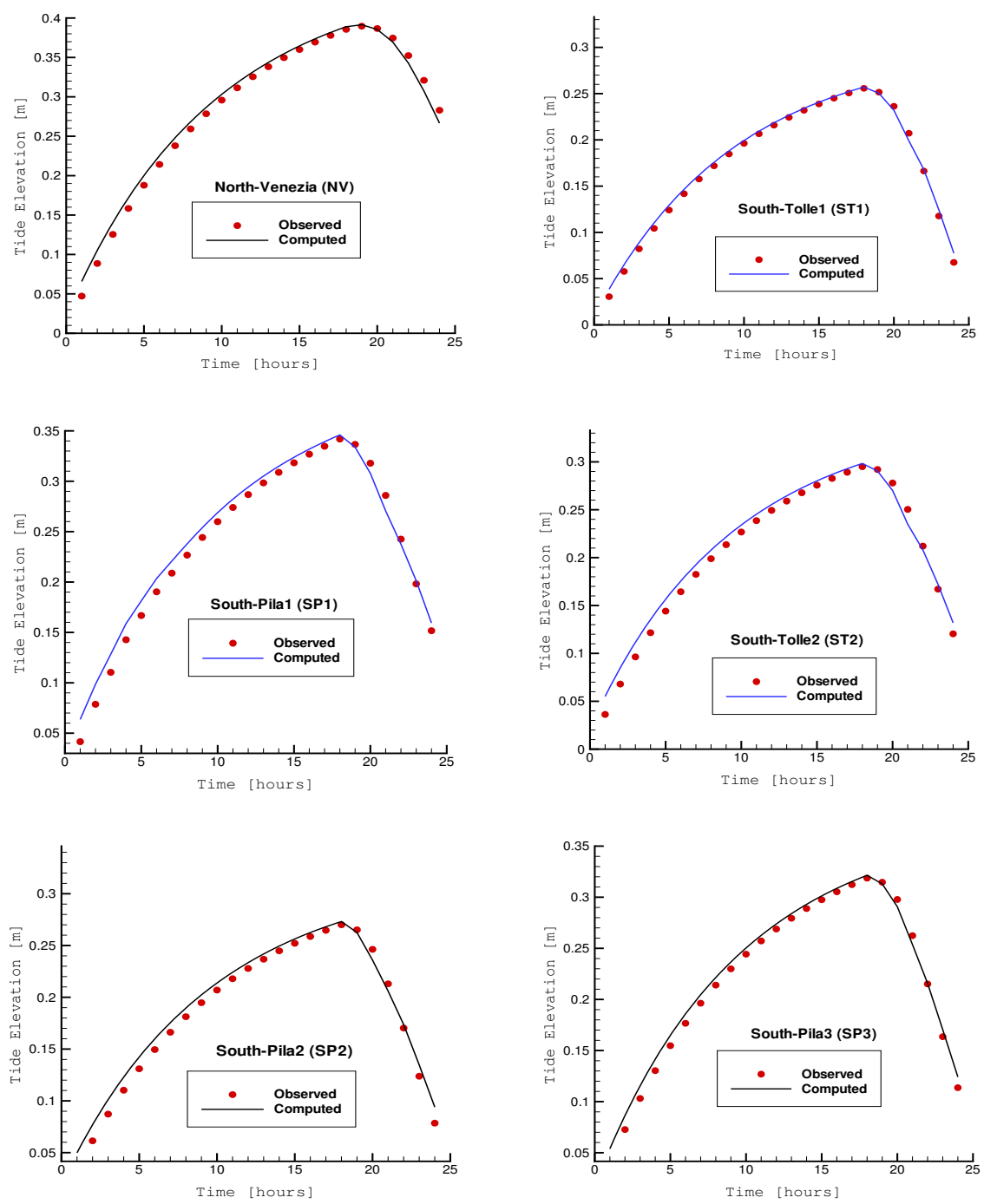

Fig. 1. Comparison of the computed and observed tidal elevations at river upstream and open sea boundaries in PRE 
In this simulation, the horizontal unstructured mesh of simulated area has been divided into 3185 triangles in each horizontal layer, and 2172 nodes with the mean space step size ranging from $d^{T}=6.2$ to $46.08[\mathrm{~m}]$ (where $d^{T}$ denotes the diameter size of the circumscribed triangle). The vertical is divided into 30 layers. To account of the boundary layer effects the bottom is divided into several thin layers of thickness $\delta z_{b}=0.5[\mathrm{~m}]$. The simulation period is from 14.00 of $1^{\text {st }}$ of May to 14:00 of $2^{\text {nd }}$ May 1999 for hydrodynamic calibration. The forcing is neap tide in wet season. There are seven tide gauges and seven tide stations and
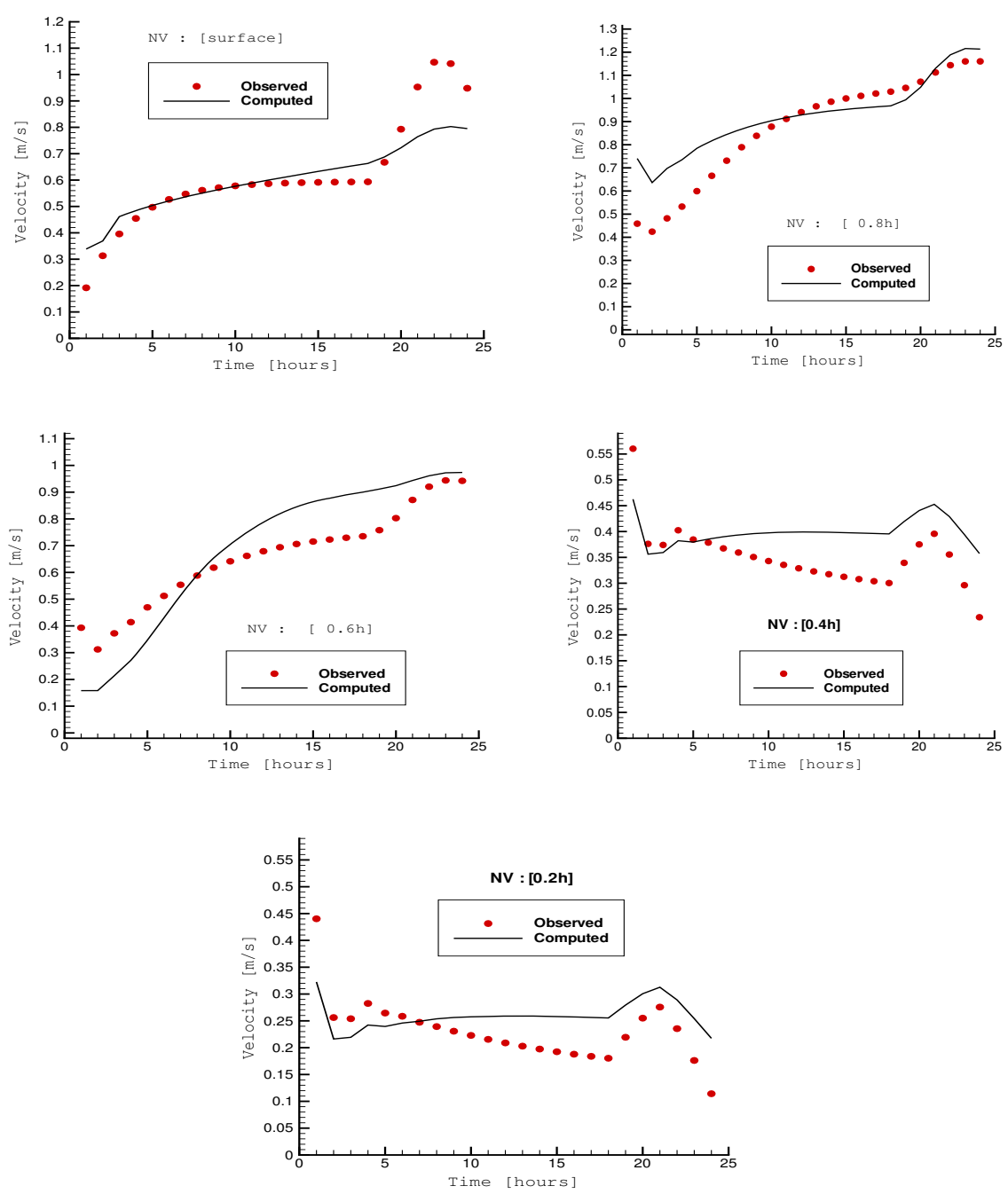

Fig. 2. Comparison of the computed and observed current velocities at North-Venezia station (NV) in PRE 
water open boundaries were controlled by tidal levels located at $N V, N T, S T 1$, $S T 2, S P 1, S P 2, S P 3$.

Figure 1 shows the comparisons of the computed and observed tidal elevations during the flood at different tide stations. As indicated, the model predicts the tidal elevations with good accuracy. Figure 2 shows the comparisons of the computed and observed flow velocity magnitude at the river upstream tide stations and different vertical layers position at respectively free-surface, $0.8 \mathrm{H}, 0.6 \mathrm{H}$, $0.4 H$ and $0.2 H$. Good agreement is found between computed and observed velocity distributions. The hydrodynamics computations have shown the ability of the model. A wet spring current was chosen for one-year simulation period from May 1999 to April 2000. The PRE is found to be a stable and fairly stratified estuary (see 4]). This is in good accordance with the field observation.

\section{Conclusions}

A Three-dimensional finite element solver has been successfully applied for simulating temporal behavior of stratified water bodies with environmental forcing, by solving the SWE for incompressible flow using the hydrostatic assumption for pressure. Processes modeled include heat and salt distribution, tidal forcing, surface wind forcing, inflows and outflows as well as the turbulence modeling for multiphasic fluid-solid flow. The effects of salinity and temperature are seen during periods of simulation, and the micro-tidal PRE (i.e. tidal elevation $<2$ $[\mathrm{m}])$ is found to be a fairly stratified estuary. The model predicts realistically the complex major features and their consequences on the $3 D$ estuarine flows. The water elevation, velocity profiles computed from the numerical models were compared successfully to the experiments. However, further application including the transport of sediment on the harbor channel is needed to improve the general applicability of the model, and this will be next stage of this work.

\section{Acknowledgment}

The first author gratefully acknowledged Funding from the Swiss National Science Foundation through grant number 21-65095.01. Alfio Quarteroni and Edie Miglio are acknowledged for their fruitfully discussions.

\section{References}

1. V. Casulli and R. T. Cheng. Semi-implicit finite difference methods for threedimensional shallow-water flow. Int. Numer. Meth. Fluids, 15:629-648, 1992.

2. K. W. Chau and Y. W. Jiang. 3d numerical model for pearl river estuary. J. Hydr. Engrg., 127:72-82, 2001.

3. Y. Chen, O. W. H. Wai, Y. S. Li, and Q. Lu. Three-dimensional numerical modeling of cohesive sediment transport by tidal current in Pearl River Estuary. Int. J. Sediment Res., 14:107-123, 1999. 
4. W. H. Graf and M. S. Altinakar. Hydraulique Fluviale, Tome II. Presses Polytechniques et Universitaires Romandes, CH-1015 Lausanne, Switzerland, 1996.

5. C. Leupi. Numerical Modeling of Cohesive Sediment Transport and Bed Morphology in Estuaries. PhD thesis, Ecole Polytechnique Fédérale de Lausanne-EPFL, No. 3266, 2005.

6. C. Leupi and M. S. Altinakar. Finite element modeling of free-surface flows with non-hydrostatic pressure and $k-\varepsilon$ turbulence model. Int. J. Numer. Meth. Fluids, page In press, 2005.

7. C. Leupi, E. Miglio, M. Altinakar, A. Quarteroni, and M. Deville. Quasi-3D finite element shallow-water flow with $k-\epsilon$ turbulence model. In Altinakar M. S., Wang S. S. Y., Holz K. P., and Kawahara M., editors, Proc. of 6th Int. Conf. HydroScience and Engrg, 6, 400-402 \& on CD-Rom, Brisbane, Australia, May 31, June 03 2004. ICHE, SWang S. Y., NCCHE, University of Mississippi, Carrier Hall, University, MS38677, USA.

8. O. Lie-Yauw and L. G. Mellor. A three-dimensional simulation of the hudsonraritan estuary. part i: Comparison with observation. J. Phys. Ocean., 15:16931709, 1985.

9. O. Lie-Yauw and L. G. Mellor. A three-dimensional simulation of the hudsonraritan estuary. part i: Description of the model and model simulations. J. Phys. Ocean., 15:1676-1692, 1985.

10. Q. M. Lu and W. H. O. Wai. An efficient operator splitting scheme for threedimensional hydrodynamics computations. Int. J. Numer. Methods Fluids, 26:771789, 1998.

11. P. J. Luyten, E. Deleesnijder, J. Ozer, and K. G. Ruddick. Presentation of a family of turbulence closure models for stratified shallow water flows and preliminary application to the Rhine outflow region. Continental Shelf Res., 16:101-130, 1996.

12. B. Mohammadi and O. Pironneau. Analysis of $k-\epsilon$ Turbulence Model. Research in Applied Mathematics. John Wiley \& Sons, Chichester, 1994. 